Student Drivers the Morning After Drinking: A Willingness to Violate Road

\title{
11 Highlights:
}

12

- A naturalistic design to investigate the morning after effects of alcohol on students' driving

- In the morning after condition drivers were more likely to violate speed limits 
31 Substantial research has investigated the effects of alcohol consumption on cognitive functions.

32 However, little research has been conducted which examines the effects of evening alcohol consumption on next morning driving performance. The current study investigated the effects of a night out involving drinking on students' morning after simulator driving performance, conducted as a within-subject naturalistic study. Thirty student drivers between the ages of 19-23 participated. Driving performance measures and eye movements were recorded while participants performed a short-simulated motorway driving task between 9-10 a.m., both after an evening consuming alcohol and on a control morning (no alcohol consumed). The task required drivers to respond to a speed limit and hazardous vehicle, with driving performance being compared over four road sections (speed reduction section, hazard section and two control sections). Sleep duration the night before the drives

41 and breath alcohol content immediately before each drive were recorded. The main findings indicate that despite the majority of drivers being legal to drive, in the morning after condition drivers tended to travel at higher maximum speeds, travel for a longer period of time over the speed limit and demonstrate a larger variance in speed. However, no differences were found in visual attention measures. These findings suggest that the morning after drinking is associated with dangerous driving behaviour in terms of violating road rules even when no deficits in attention are observed. The implications for road safety are discussed, focusing on informative programmes to educate drivers of the dangers associated with morning after driving. 
56 From past research it is evident that driving performance is impaired by alcohol even in low dosage 57 (Ogden \& Moskowitz, 2004; Weafer \& Fillmore, 2012), with alcohol usage in the general night 58 driving population corresponding to increases in alcohol related crashes (Carlson, 1972). Alcohol 59 consumption results in a particularly high risk of road crashes and fatalities that are reflected in national statistics such as the finding that in $2016,13 \%$ of all fatalities on roads in the UK were associated with alcohol consumption (DfT, 2017a).

The impairments that occur after the consumption of alcohol impact a number of cognitive functions including motor control, planning, tracking, attention, and psychomotor performance (Fogarty \& Vogel-Sprott, 2002). Driving, a complex cognitive task, has been shown to be impaired by alcohol across a number of measures including lateral vehicle control such as standard deviation of lateral position (SDLP) (Mets et al., 2011, Verster \& Roth, 2014) and longitudinal measures including mean speed (Mets et al., 2011; McCartney, Desbrow, \& Irwin, 2017), and standard deviation of speed (SDSP) (Marczinski, Harrison, \& Fillmore, 2008). In a meta-analysis and systematic review of seventeen studies investigating the effects of alcohol on simulated driving, Irwin et al. (2017) identified that both SDSP and SDLP are the two most sensitive indicators of driving impairment however, earlier research indicated that SDLP was the most stable and sensitive measure of detecting driving impairment from alcohol intake (Roth \& Verster, 2011; Verster \& Roth, 2014). The driver's ability to maintain a stable lane position and maintain a safe speed are imperative to driver safety, since greater deviation increases chances of crossing into the paths of other vehicles or drifting off the road (Verster \& Roth, 2014), as well as speed variance influencing crash risk and crash severity (Reed \& Green, 1999). Additionally, alcohol has also been seen to have sedative properties, where it has been proposed that it may initiate disinhibitory behaviours. This had been thought to explain risky driving impairments caused by alcohol including unnecessary lane changes, speeding, and ignoring traffic signals (Fillmore et al., 2008; Weafer \& Fillmore, 2016). 
While the immediate deleterious effects of alcohol on cognitive performance are reasonably well quantified, relatively few studies have investigated the 'morning after' detriment that previous alcohol consumption may have on everyday activities such as driving related skills and abilities (Stephens, 2008; Ling et al., 2010). This may be particularly important since reviews concerning traffic accidents at different times of day have found early morning driving to be particularly dangerous even when accidents due to alcohol are removed from the dataset (Akerstedt, Kecklund \& Hörte, 2001).

\subsection{The Morning After Effects of Alcohol on Driving}

The alcohol hangover is the most commonly reported symptom of heavy drinking the night before. However, there has been dispute as to whether hangovers begin at a low declining blood alcohol concentration (BAC) or when reaching zero (Verster et al., 2010). There is now consensus regarding the definition for alcohol hangover, with an alcohol hangover referring to a combination of mental and physical symptoms experienced the day after an episode of excess drinking, starting when BAC approaches zero (van Schrojenstein Lantman et al., 2016; Verster et al., 2017). Previous research has suggested that there is a biphasic effect in cognitive impairments as a function of the BAC, forming an inverted-U- shaped curve (Starkey \& Charlton, 2014; Charlton \& Starkey, 2015). Specific BACs on the increasing limb of this curve may have different behavioural effects to equivalent BACs measured a few hours later on the decreasing limb of the same curve. As individuals' BACs move on the descending limb of the BAC curve, motor coordination is known to recover more quickly than cognitive abilities. Contextually, this may result in drivers perceiving an improvement in visuomotor skills and a feeling of reduced intoxication while being unknowingly still impaired (Cromer, Cromer, Maruff, \& Snyder, 2010; Marczinski \& Fillmore, 2009). Charlton and Starkey (2013) describe the impairments that continue to be observed on the descending limb as acute protracted errors (APEs), which can be seen in participants in driving at higher maximum speeds and producing a greater number of edge line crossings when participants' BAC had reached a level on the descending limb 
compared to the same BAC on the ascending limb. The descending limb of the BAC curve is of particular importance as it is likely that decisions to drive are made when drivers are in this state which can often persist to the beginnings of a hangover (Weafer \& Fillmore, 2016). Previous research has found that the effects of an alcohol hangover on general cognitive tasks are larger for participants in hungover conditions compared to individuals who were still intoxicated (McKinney et al., 2012). However, to contextualise this research it is imperative that researchers use actual driving tasks as opposed to tests on general cognitive abilities to understand next day effects of alcohol.

One of the few studies focusing on the effects of a hangover on driving involved volunteers aged 2246 and found that acute intoxication severely impaired driving performance but to a much lesser degree the following morning (Törnros \& Laurell, 1991). This study was conducted in a driving simulator, however, it is not clear to what extent these tests related to normal driving, as participants were asked to complete the drive in as short time as possible, resembling race track driving more than typical driving. More recent evidence from Verster et al. (2014a) involved a one-hour highway simulated drive comparing the impairments in driving behaviour after drinking the night before and a control day. Results found that although blood alcohol concentrations (BAC) was zero, driving was significantly impaired when participants were hungover relative to the control drive for lateral measures of driving, exhibited through increased SDLP as well as driving being self-reported as significantly poorer by the participants. The researchers found no differences in longitudinal measures such as standard deviation of speed (SDSP), implicating impaired attention as the fundamental mechanism to explain the observed effects. This one-hour simulated drive is representative of highway driving which requires sustained attention and vigilance, however, this task is in contrast to the most common driving routes in the UK in terms of the driving task and task length. The 2016 National Travel Survey reported the average duration per car driver trip as 22 minutes (DFT, 2017a), with the majority of these journeys requiring city driving. 
132 In addition to these findings, Verster et al. (2014b), gave reason to believe that driving the morning 133 after alcohol consumption is a growing concern. From interviewers of 343 professional Dutch truck 134 drivers, it was found that more than half admitted to driving with a hangover (56.4\%), and felt their 135 driving was significantly worse compared to no alcohol days. This illustrates that driving with a

136 hangover may be more common than initially expected and therefore, experimental research needs to 137 further investigate the consequences of morning after driving on on-road performance.

\subsection{The Morning After Effects of Sleep deprivation and Alcohol on Driving}

140 Sleepiness is not only known to independently cause impairments equivalent to that of alcohol 141 (Williamson \& Feyer, 2000), but has also been found to exacerbate the negative effects of alcohol 142 (Banks et al., 2004) as well as cause engagement in risky behaviours (Orzel- Gryglewska, 2010). A 143 more recent study has found that reduced sleep is associated with more severe alcohol hangovers (van 144 Schrojenstein Lantman et al., 2017). Sleepiness has been seen to seriously impair driving performance however, unlike the effects of alcohol which can be observed after a small consumption, changes in behaviour as a result of sleepiness have only been reported when high levels of fatigue are reached (Ingre et al., 2006), with much research investigating prolonged wakefulness rather than acute sleep deprivation. From the few studies that have investigated the effect of partial sleep deprivation on

149 participants' driving performance, it has been found that sleep deprivation significantly affects driving measures such as standard deviation of lateral position (SDLP) in both a simulation environment

151 (Otmani et al., 2005) and on-road (Jongen et al., 2015), mean speed (Peters et al., 1999), as well as causing a narrowing of visual search, hindering the processing of peripheral signals (Rogé et al., 153 2003).

154 The most commonly reported measure impaired from fatigue is the ability to maintain appropriate lateral position (SDLP) (O’Hanlon \& Kelly, 1974; Ingre et al., 2006). Theoretically, this appears to associate impairment through fatigue with inattention which has been argued to be the most important 
lane position is a particularly sensitive measure of driver fatigue as it requires the highest degree of

159 steadiness, and fatigue is known to slow motor functions and reactions, both of which reduce the 160 ability to maintain lane position (Ingre et al., 2006). This slowing in functions has also been found to 161 lead to diminished processing of information which can impair responses to hazardous situations 162 (Gharagozlou et al., 2015), indicating that sleep deprivation can lead to dangerous impairments in 163 driving by affecting both sustained attention and vigilance.

164 Measures of drivers' eye movements can provide useful insight into the allocation of attention and 165 vigilance (Deubel \& Schneider, 1996; Velichkovsky et al., 2002). Eye movements and fatigue have been previously investigated, with fatigued participants having been reported to exhibit narrower gaze as a result of reduced vigilance leading to impaired information processing (Ji \& Yang, 2002). Eye movements have also been seen to be affected by alcohol, however, this has not been as extensively researched. Ogden and Moskowitz (2004) state that alcohol causes a slowing in the processing of surrounding information, resulting in longer fixations on objects in order to perceive them.

171 Consequently, effects of alcohol and sleep deprivation on eye movements may elicit similar impairments in attention and together culminate in a potentially dangerous combination on the road.

Some research has been conducted observing the combined effects of both prolonged wakefulness and alcohol consumption on simulated driving performance. Social drinking is often associated with individuals going to bed later than normal, so in normal contexts sleepiness may routinely exacerbate these physiological effects of recent alcohol consumption (Hershner \& Chervin, 2014). Arnedt et al., 178 (2000) compared measures of subjective sleepiness, simulated driving performance and drivers' ability 179 to judge impairment. Subjective sleepiness was measured using the Stanford Sleepiness Scale, before 180 and after each driving session. Findings suggested that the combination of prolonged wakefulness and 181 alcohol consumption produced greater impairment in simulated driving performance than each factor alone, increasing SDLP and SDSP. Performance was also seen to decrease with time-on-task. 


\subsection{Naturalistic Studies}

Many of the previously discussed studies investigating the effects of alcohol consumption and sleep deviation on driving performance have used laboratory based experimental designs, whereby these parameters have been systematically counterbalanced. This drinking experience contrasts with natural drinking environments (Brookhuis, 2004), and previous research has suggested that no constraints on behaviour is best practice for studying next day effects of alcohol (Prat et al., 2008). For this reason, research efforts have been directed towards investigating the effects of alcohol in naturalistic settings, investigating cognitive functioning in a social setting where alcohol consumption is self-regulated. Degia et al., (2006) found that when using portable testing equipment at music festivals, alcohol impaired both divided and sustained attention. Moreover, Tiplady and Degia (2004) found overall impaired performance on a handheld forced choice device, which is thought to measure attention, working memory and size estimation; a tester used for detecting driver impairment on the roadside. This device has been seen to be sensitive to the effects of alcohol both in natural settings and in the lab (Tiplady et al., 2005).

These naturalistic studies have been furthered by investigating the effects of a naturally occurring 201 hangover, when the consumption of alcohol has been under personal control (McKinney and Coyle, 2004). These studies have revealed that under naturalistic conditions, participants' performance is still impaired the morning after alcohol consumption, with this being demonstrated in cognitive functions such as memory, psychomotor performance, and attention when using tasks such as free recall and selective attention tests (McKinney and Coyle, 2004), speed and capacity language processing tests 206 (Finnigan et al., 2005), and mood and anxiety tests on students (McKinney and Coyle, 2005). As 207 aforementioned, one of the only studies to investigate the morning after effect of alcohol consumption on driving directly, also used a naturalistic study design (Verster et al., 2014a). 


\subsection{The Student Population}

211 University students are a population at risk of binge drinking behaviour and exceeding weekly

212 consumption guidelines (e.g. 14 units in the UK; NHS, 2018). Recently, a large-scale Students and

213 Alcohol Survey conducted by National Union of Students (NUS) revealed that $79 \%$ of students

214 ( $\mathrm{n}=1240)$ agreed that drinking and getting drunk is part of university culture (NUS, 2017). This

215 behaviour is of particular concern to the transport industry since intoxicated driving is renowned for

216 having fatal consequences (World Health Organisation, 2016). Encouragingly, the number of fatal or

217 serious crashes involving young drivers caused by drink driving has decreased significantly over the

218 last 14 years in the UK, with 450 young drivers (17-24 years old) involved in a fatal crash while over

219 the alcohol limit in 2001 compared to 170 in 2015 (DfT, 2017b). However, there is concern that a

220 considerable amount of drink driving or driving while still impaired from the effects of alcohol may be

221 occurring unrecorded (MacDonald, 1999). Moreover, for young drivers in particular, sleep deprivation

222 has been found to increase risk of crashing, evident in one prospective study on more than nineteen

223 thousand 17 to 24-year-old drivers in Australia, which found that those who sleep six or fewer hours

224 were more likely to crash, even when driving exposure was controlled (Martiniuk et al., 2013).

225 Previous research has also found that young drivers' willingness to drive under the influence of

226 alcohol is high, particularly with young drivers who often binge drink. It has been found that binge

227 drinkers report less subjective sleepiness and convey a greater perceived ability to drive compared with

228 non-binge drinkers, despite all participants showing greater difficulty maintaining lane position and

229 appropriate speed in addition to making multiple driving errors when intoxicated compared to

230 performance in a placebo condition (Marczinski, Harrison \& Fillmore, 2008). The Royal Automobile

231 Club (RAC) motoring report 2017 revealed that, out of a representative sample of 1700 drivers, $16 \%$

232 of motorists admitted to drink-driving, and $10 \%$ of motorists believed it occurred as a result of still

233 being over the limit the morning after drinking (RAC, 2017). The RAC estimate that this could equate

234 to five million motorists nationally who think they have driven over the limit, with the majority 
235 believing it occurred the morning after. Since undergraduate students are likely to have morning 236 university commitments following a commonly occurring night out, there is potential that they may 237 constitute a significant part of the RAC's $10 \%$ of people driving in the morning over the limit.

\subsection{The Current Study}

Driving impairments from alcohol consumption and sleep deprivation are similarly natured,

241 particularly apparent in terms of diminished vigilance and attention, and have been found to persist to 242 the morning after (Verster et al., 2014a). The present study had an ecologically representative testing 243 protocol, with no constraints posed on the participants' drinking and sleeping behaviour. This involved monitoring a sample of students consuming their usual type of alcoholic beverage in their chosen social situation. As many factors can influence morning after impairments such as physical activity during the night out, drinking rate, venue and smoking, it is extremely difficult to control all of these factors and therefore it is questionable to what extent controlled procedures measure realistic effects. In contrast, a naturalistic design allows participants to consume alcohol unsupervised, with no constraints on the amount of alcoholic drinks and type of beverage as well as sleeping in their own environment of choice. This provides the opportunity to assess the likely impacts of typical social drinking with the potentially associated sleep disruption in a student sample on their normal driving behaviour the morning after.

The present study focuses on driving performance in a young student population between the ages of 19-23, the morning after a university night out. Previous research and crash statistics suggest that this is a high-risk target group and a sample who would be expected to engage in early morning university commitments, which may involve the need to drive. The current study fills a gap in the literature as it is the first to explore the morning after effects of students' unrestricted social drinking on simulated driving performance compared to the morning after a control night with normal sleep and no alcohol consumption. 
263 The current naturalistic study was conducted to investigate whether undergraduate students were 264 impaired at simulator driving the morning after a night out social drinking, compared to a control 265 night. Based on previous research, it is predicted that driving will be significantly impaired the 266 morning after compared to a control day (no alcohol consumed). It is predicted that the morning after 267 condition will adopt significantly higher mean speeds, higher maximum speeds, break the speed limit 268 for a higher percentage of time, and have a larger SDSP and SDLP compared to the control condition. 269 It is also predicted that participants' response time to everyday driving hazards will be significantly 270 slower than in the control condition. Finally, it is predicted that drivers will have narrower visual 271 attention in morning after condition, demonstrated by reduced standard deviation of horizontal fixation 272 locations and vertical fixation locations, as well as longer mean fixation durations.

\section{Methods.}

\subsection{Design}

The experiment used a within subjects design with all participants completing a simulated motorway drive the 'morning after' a night of social drinking and a control drive, after a night involving no alcohol consumption. These conditions were counterbalanced to control for carry over effects of a repeated task, with 15 participants having completed the first drive the 'morning after' and 15 of the participants completing the control drive first.

The experimental motorway drives were split into 8 road sections, shown in Table 1 below. For the analysis, 4 road sections of equal length were used, each approximately $1000 \mathrm{~m}$ in distance. The 4 road sections not used for analysis served as an initial practice drive and padded out the road sections of 
interest. The first section was 'Control Section 1', where participants were able to drive along the motorway at an instructed speed of $65 \mathrm{mph}$. The second 'Speed Reduction Section' began when an overhead gantry sign was in sight, instructing participants to slow down to $40 \mathrm{mph}$ due to roadworks ahead. This section ended when a delimit gantry was in sight, allowing participants to return to 65 mph. The third road section, 'Control Section 2', allowed participants to return to their normal motorway speed of around 65mph. Finally, the fourth 'Hazard Section' began when a vehicle situated on the hard shoulder was in sight (either a motorbike or car). This vehicle pulled out into the path of the participant's vehicle as they approached.

Table 1: Shows the 8 road sections of the motorway drive and their distances. Road sections in white were the 4 equal sections used for analysis and the grey sections were distances driven before and between sections that were not used in the main analysis.

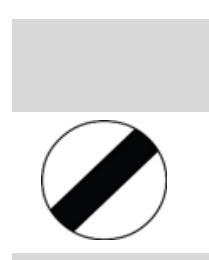

Start of Drive - Control Section 1 Start

$5407 \mathrm{~m}$

Control Section 1

$1000 \mathrm{~m}$

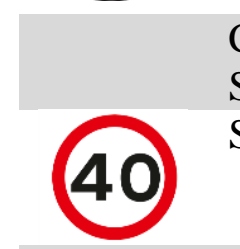

Control section 1 End - Speed Reduction Section $201 \mathrm{~m}$

Start

Speed Reduction Section

$1000 \mathrm{~m}$

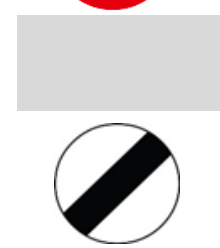

Speed Reduction Section End- Control Section $2 \quad 23$ m

Start

Control Section 2

$1000 \mathrm{~m}$

Control Section 2 End- Hazard Section Start

$191 \mathrm{~m}$ 


\subsection{Participants}

314 Full ethical approval was granted prior to conducting the experiment by the University of Nottingham 315 Psychology Ethics Committee. A power analysis was conducted using the software package, G*Power 3163.1 (Buchner, Faul \& Erdfelder, 1992). The overall sample size of 30 was enough to provide more than 317 adequate power $(>.99)$ to detect a large within-subjects effect $(d=.8)$ or provide moderate power (> $318.75)$ to detect a medium effect size $(d=.5)$. Thirty participants completed both the morning after and 319 control drives: 10 males and 20 females, with ages ranging from 19 to 23 years $(M=20.9, S D=1.0)$. 320 All participants were recruited through advertisement at the University of Nottingham and received a $321 £ 10$ inconvenience allowance. All participants were students who were known to be socialising the night before the experimental condition, enough to assume a next morning alcohol hangover. All participants had a full British driving licence, with years licenced ranging from 1-6 years $(M=3.33$, $S D=1.40)$, with their annual mileage ranging from $0-10000$ miles $(M=3133.33, S D=2747.83)$. An additional two participants completed a screening drive, however, as they reported symptoms of simulator sickness during screening they were not included in the main experiment.

\subsection{Materials/Apparatus}

Due to the experiment having multiple stages, participants had to complete a separate information sheet and consent form for the screening phase and the experimental drives. The screening phase information sheet explicitly explained the purpose of this stage and, importantly, if they felt any discomfort in the driving simulator they should end the experiment immediately. The information sheet for the experimental drives explicitly explained what the driving task involved, stating two important specific requirements that participants had to adhere to when taking part in this experiment. Firstly, it highlighted that participants should not drive or cycle to or from the experiment due to potential alcohol impairment. Secondly, it required that participants did not drive for 30 minutes after taking part in any phase of the experiment due to the simulation environment having potential carry- 
over effects on driving on real roads. Standard simulator sickness questionnaires (Kennedy et al., 1993) were filled out by participants before and after the screening drive and the two experimental drives to check for signs of simulator sickness.

Participants completed an 'Alcohol Consumption and Sleep Questionnaire' prior to both experimental drives which included self-reported sleep duration, units of alcohol consumed the night before and time of their first and last drink. Subjective sleepiness was also scored using the Stanford Sleepiness Scale 'Alertness Test' (Shahid, Wilkinson, Marcu \& Shapiro, 2012), which was also completed prior to each drive. This was a self-rating scale which was used to quantify sleepiness, comprising of a seven-point Likert-type scale which had descriptors ranging from "feeling active, vital alert, or wide awake" (score = 1) to "no longer fighting sleep, sleep onset soon and having dream-like thoughts" $($ score $=7)$.

Prior to each drive, a Dräger Alcotest 6810 breathalyser device (Dräger Safety, Germany) was used to measure participants' breath alcohol concentration (BrAC). The breath alcohol level limit in England is 35 microgrammes of alcohol per 100 millilitres of breath, as this has been seen to increase reaction times and decrease attention (Grant et al., 2000). In addition, the night before each experimental drive, sleep duration was measured objectively using an actigraphy Fitbit flex activity tracker. This device was worn on the wrist of the participant to track movement while sleeping, with software translating these movements into periods of sleep and wake, outputting actual sleep duration in hours and minutes.

The driving scenarios were performed using Nottingham Integrated Transport and Environment simulation (NITES) facility's medium fidelity, fixed based driving simulator (NITES 2). The simulator consisted of a five-metre diameter hemi-cylindrical screen, subtending 180-degrees of the visual field, and included a rear display screen on a 36-inch LCD television. The fixed based driving rig consisted of a force feedback steering system, adjustable seat, and a dashboard that included a steering wheel 
and speedometer. There was also a gear lever, brake, clutch and accelerator pedal for vehicle control.

365 The rig was faced centrally towards the 180 degrees of visual field. The driving scenarios are formed 366 on the screen using three projectors, allowing the driver to see the road from the driver's perspective.

367 The scenarios are run using XPI simulation software (XPI Simulation, London, UK), generating

368 realistic roadway scenery. The experimental motorway drive aimed to resemble a real-world motorway 369 scenario. Participants' eye movements were also tracked continuously throughout their drives, using 370 two linked FaceLAB 5.0 eye tracking systems (four cameras and two infrared sources), allowing 371 participants' eye movements to be tracked continuously over a range of approximately 90 degrees of 372 forward visual angle.

\subsection{Procedure}

Participants were approached to take part in the study around a week before the intended night out. Up to a week before the first experimental drive, participants were asked to partake in a screening drive to check for any signs of simulator sickness. This stage consisted of a short drive that lasted around 5 minutes following a route that contained both straight roads and corners. A simulator sickness questionnaire was completed before and after the screening drive to look for any significant changes on any of the items. Participants who did not suffer from simulator sickness were asked to take part in the main experimental study.

At this point, participants with no signs of simulator sickness were given a second information sheet and consent form explaining what the main study involved and the necessary information regarding the sleep tracker. Participants were then given the Fitbit flex activity tracker and told to wear this device until they returned the next morning. It was also requested that participants keep track of roughly what time they went to bed and awoke the following morning. For careful counterbalancing, fifteen of the 
participants completed their first motorway drive after a night of socialising, while fifteen of the participants took part in their first drive after a control night without socialising.

For the main task, participants visited NITES 2 between 9 and 10 a.m. the following morning after either the control or socialising night. Firstly, all participants were breathalysed, recording their BrAC readings. They were then asked to fill out the 'Stanford Sleepiness Scale' and 'Alcohol Consumption and Sleep Questionnaire' before the drive. In addition, participants were also asked to fill out a simulator sickness questionnaire before and after the drive.

Participants then made themselves comfortable in the simulator and the eye trackers were calibrated for each individual to record their eye movements. The experimenter read out the following systematic instructions to each participant before each drive; 'When completing the drive, you should keep in the left-hand lane whenever possible at a speed of $65 \mathrm{mph}$, unless instructed otherwise'. Following these instructions, participants then completed a motorway drive that lasted around 15 minutes. A motorway drive was purposely chosen as this should have been a fairly easy task for all participants.

Each drive contained two hazards. In both drives, the motorway contained a 'Speed Reduction

Section' which was indicted by an overhead gantry instructing participants to slow down to $40 \mathrm{mph}$ due to roadworks ahead. Later participants encountered a delimit sign on a gantry indicating that they were able to return to normal speed. A second hazard was designed to surprise participants and cause them to brake or swerve to avoid a collision. Depending on the counterbalanced condition, in the first drive a car parked in the hard shoulder pulled out in front of the participant's vehicle. In the second 411 drive at the exact same point, a motorbike pulled out of the hard shoulder in front of the vehicle. The 412 opposite order applied for half of the participants. From the point at which the hazard was first visible, 413 the participant's time to respond to the hazard was measured. 
415 Participants returned within a week to complete the second drive. The fifteen participants who 416 completed their first drive after a control day then went out socialising, while the other fifteen did not 417 and then participated in their control drive. The same procedure was carried out for the second drive. 418 At the end of all sessions, participants were given their inconvenience allowance and a debrief 419 document to take away with them.

\section{Results.}

In terms of the criterion for reporting findings, all significant results ( $p<.05$, two-tailed) will be reported. Where non-significant results represent at least a medium effect size $\left(d>=0.5,{ }_{\mathrm{p}} \mathrm{n}^{2}>=0.09\right)$ or are theoretically important they will be included in square brackets []. Where within subjects factors include three levels, Mauchly's test of sphericity has been used and in cases where a significant breach of sphericity was detected significance testing has been conducted using Greenhouse-Geisser adjusted degrees of freedom. Occasions where this has been done are indicted by the use of decimal places in the reported degrees of freedom.

\subsection{Objective Sleep Actigraphy Measures}

All thirty participants' sleep data were analysed. A paired samples t- tests was conducted to test for significant differences in sleep duration between the Control and Morning After condition. Sleep

433 Duration was significantly lower in the Morning After condition ( $M=4.63$ hours, $\mathrm{SD}=1.21)$, compared to the Control condition $(\mathrm{M}=7.32$ hours, $\mathrm{SD}=1.00)\left(t(29)=9.87 ; p^{<.001,} d=1.79\right)$.

\subsection{Subjective Sleep Measures}

437 The majority of participants disclosed their average hours slept a night to be between 6-8 hours. In 438 terms of their subjective self-report sleep duration on the two experimental nights, this was 
significantly lower in the Morning After condition $(\mathrm{M}=4.81, \mathrm{SD}=1.21)$, compared to the control condition $(\mathrm{M}=7.59, \mathrm{SD}=1.16)(t(29)=9.20 ; p<.001, d=1.68)$.

Subjective sleepiness on the Stanford Sleepiness scale was significantly higher in the Morning After condition $(\mathrm{M}=4.33, \mathrm{SD}=1.15)$, compared to the Control condition $(\mathrm{M}=2.03, \mathrm{SD}=.85)(t(15)=8.60$; $p<.001, d=1.58)$.

\subsection{BrAC and Subjective Alcohol Consumption}

In terms of measurable $\mathrm{BrAC}$ at the time of testing, 28 participants were under the legal $\mathrm{BrAC}$ limits in both conditions (legal limits=35 microgrammes of alcohol per 100 milliliters of breath), whereas 2 participants in the morning after condition were over this limit, with readings of 52 and 54. All participants had a BrAC reading of 0 in the Control condition. In the Morning After condition readings ranged from 0 to $54(\mathrm{~m}=9.57)$.

In regards to self-reported units of consumed alcohol, these are reported against BrAC readings for each condition in Table 2 below. It is immediately obvious that there is no simple relationship between subjective units of alcohol consumed and BrAC for the self-reports of between 8 and 20 units. Although the five participants with the lowest self-reported alcohol consumptions did indeed have no breath alcohol at the time of testing, after this the relationship breaks down. From the seven participants who reported drinking between 8-12 units the evening before, their breathalyser reading ranged from $0-28$, providing an average of 11.9 . This was extremely high compared to greater subjective unit categories of 12-16 and 16 to 20, however, again the 8 participants who self-reported drinking the most did have some of the highest breath alcohol readings, including the two participants who were over the legal limit.

$\underline{\text { Table 2: }}$ : The number of participants who fall into each of the unit categories in terms of self-reported number of units of alcohol consumed for both conditions, as well as the mean BrAC for those participants in each of the unit categories. 
Condition

\begin{tabular}{|c|c|c|c|c|}
\hline & \multicolumn{2}{|c|}{ Control } & \multicolumn{2}{|c|}{ Morning After } \\
\hline Self-Reported Units of & & Mean Breath & & Mean Breath \\
\hline Alcohol Consumed & $n$ & Alcohol & $n$ & Alcohol \\
\hline $0-4$ & 30 & 0 & 0 & - \\
\hline $4-8$ & - & - & 5 & 0 \\
\hline $8-12$ & - & - & 7 & 11.9 \\
\hline $12-16$ & - & - & 5 & 3.6 \\
\hline $16-20$ & - & - & 5 & 5.2 \\
\hline $20+$ & - & - & 8 & 20.0 \\
\hline
\end{tabular}

465 A multiple logistic regression was conducted with the outcome variable of objective BrAC for the 466 morning after condition, using the predictor variables of objective sleep duration, subjective sleep 467 duration, Stanford Sleepiness Scale ratings (SSS), subjective alcohol consumption and reported time of 468 last drink. For BrAC is was found that by adding five key predictor variables (the four above, plus 469 objective sleep duration), a significant regression equation was found $(\mathrm{F}(5,29)=5.074, p<.01)$, with 470 an $\mathrm{R}^{2}$ of .514. The significant contributions to the model were made by SSS (p<.01) and time of last 471 drink $(\mathrm{p}<.05)$. It was found that participants who had their last drink later in the evening, and reported 472 more subjective sleepiness on the Stanford Sleepiness Scale had more residual breath alcohol content 473 at the time of testing.

\subsection{Driving Performance}

In terms of analysing driving performance, a $2 \times 3$ repeated measures ANOVA was conducted on each 
Section 1, Speed Reduction Section and Control Section 2). For the factor of Road Section two a priori orthogonal contrasts have been specified that first compare behaviour in the Speed Reduction Section with that in the other two control road sections to assess any overall effect of the new speed limit, and secondly compare performance in Control Section 1 with performance in Control Section 2 to assess any overall changes in performance over time. The fourth 'Hazard Section' was analysed separately with paired samples t-tests comparing general driving behaviour between the Control and Morning After conditions and including additional measures to assess participants' hazard reaction times. All 30 participants' road data were analysed.

Six dependent measures were used to characterise driving behaviour - mean speed, maximum speed, percentage of time spent of the speed limit (70mph), standard deviation of speed (SDSP), mean absolute acceleration and standard deviation of lane position (SDLP). Mean speed (mph) was the average speed of drivers over each road section. Maximum speed (mph) was the highest speed each participant reached in each road section. The percentage of time spent over the speed limit was based on the total time in each road section that exceeded the motorway speed limit of 70mph. SDSP (mph) was the chosen measure of speed variance which provides an overall measure of variance within each road section. To provide a measure of the sharpness of this change, change in speed between successive samples in each road section was calculated and the absolute value of this change (acceleration or deceleration) was averaged over the road section, calculated as mean acceleration. Finally, in regards to SDLP this was calculated as the square root of the lateral position variance of the driving simulator vehicle in the four road sections.

For mean speed, there was a significant main effect of Road Section, $F(1.569,45.508)=9.014, p<.001$, $\mathrm{p}^{2}=.237$, see Figure 1a, with contrasts showing speeds being significantly lower in the speed reduction section compared to the control sections, $F(1,29)=11.644, p<.01, \mathrm{p}^{2}=.286$. 
For maximum speeds, there was a significant main effect of Condition, $F(1,29)=4.925, p<.05,{ }_{\mathrm{p}}{ }^{2}=$ .145 , indicating that drivers travelled at high maximum speeds in the morning after condition $(\mathrm{m}=68.84 \mathrm{mph})$ compared to the control condition $(\mathrm{m}=67.05 \mathrm{mph})$, see Figure 1b. For the hazard section, the paired samples t-test found that there was a marginal tendency have higher maximum speeds morning after condition $(\mathrm{m}=71.02 \mathrm{mph})$, compared to the control condition $(\mathrm{m}=68.58 \mathrm{mph})[t$ $(19)=1.769 ; p=.087, d=0.32]$.

For percentage of time spent over the speed limit, there was a main effect of Condition, $F(1,29)$ $=7.875, p<.01, \mathrm{p}^{2}=.214$, indicating that drivers spend a larger percentage of time over the speed limit in the morning after condition $(\mathrm{m}=15.70 \%)$ compared to in the control condition $(\mathrm{m}=5.69 \%)$. For the hazard section, the paired samples t-test found that the percentage of time over the speed limit was significantly higher in the morning after condition $(\mathrm{m}=21.63 \%)$, compared to the control condition $(\mathrm{m}=11.09 \%)(t(29)=2.270 ; p<.05, d=0.41)$, see Figure 1c.

In regards to SDSP, there was a significant effect of Condition, $F(1,29)=5.117, p<.05,{ }_{\mathrm{p}} \mathrm{n}^{2}=.150$, indicating that drivers had a larger speed variance in the morning after condition $(\mathrm{m}=3.46 \mathrm{mph})$ compared to the control condition $(\mathrm{m}=2.72 \mathrm{mph})$. There was a significant main effect of Road Section, $F(1.331,38.606)=15.695, p<.001, \mathrm{Pn}^{2}=.351$, with contrasts revealing that speed variance was higher in the speed reduction section than the other two control sections, $F(1,29)=17.227, p<.001, \mathrm{p}^{2}=.373$, and a significant increase in variance from the first control section to the second control section, $F$ $(1,29)=7.503, p<.05,{ }_{\mathrm{p}} \mathrm{n}^{2}=.206$, See Figure $1 \mathrm{~d}$.

For absolute mean acceleration $\left(\mathrm{ms}^{-2}\right)$ there was a marginal main effect of Condition $[F(1,29)=3.590$, $\left.p=.068, \mathrm{p}^{2}=.110\right]$ indicating that there was a sharper change in speed in the morning after condition $(\mathrm{m}=.365)$ compared to the control condition $(\mathrm{m}=.295)$. There was also a main effect of Road Section, $F$ $(1.209,35.048)=6.226, p<.05, \mathrm{p}^{2}=.177$, with contrasts revealing an overall increase in absolute 
530

531

532

acceleration from the first control section to the second, $F(1,29)=12.685, p<.001,{ }_{\mathrm{p}} \mathrm{n}^{2}=.304$, and an increase in the speed reduction section compared to the two control sections, $F(1,29)=5.483, p<.05$, $\mathrm{p} \mathrm{n}^{2}=.159$, See Figure 1e.

In regards to SDLP, there was a significant main effect of Road Section, $F(2,58)=337.687$, p <.001, $\mathrm{p}^{2}=.921$, with contrasts showing an overall increase in SDLP from the first control section to the second, $F(1,29)=703.902, p<.001, \mathrm{p}^{2}=.960$, and a reduction in SDLP in the speed reduction section compared to the two control sections, $F(1,29)=95.688, p<.001,{ }_{\mathrm{p}}{ }^{2}=.767$, see Figure $1 \mathrm{f}$.

Participants' response times to the hazard were also analysed. The criterion used to determine response time was the time from the start of the 'Hazard Section' before the participant either swerved at a rate of $1 \mathrm{~ms}^{-2}$ or decelerated at a rate of at least $1 \mathrm{~ms}^{-2}$. These criteria were chosen post-hoc to best quantify the behaviour of participants and provided plausible response time measures for all participants. A paired samples t-test found that response time was not significantly different between the two conditions $[t(29)=.62 ; p=.54, d=0.11]$.

Multiple logistic regressions were also conducted with the six driving measures as outcome variables, using the predictor variables of objective sleep duration, subjective sleep duration, Stanford Sleepiness Scale ratings (SSS), BrAC, subjective alcohol content and reported time of last drink. It was found that on all driving measures these predictor variables did not make significant contributions to the models therefore, although there was a genuine impairment in the morning after condition on some driving measures, these impairments were not predicted by these variables or a combination of these variables.

\subsection{Eye Tracking Measures}

Due to difficulties in calibration with the simulators' eye tracking system not all participants had complete eye tracking data for both drives. Eye movement data were thus analysed from the 22 
556 participants who had good eye-tracking data available for both of their two drives. The three eye 557 movement measures of interest were standard deviation of horizontal fixation locations, standard 558 deviation of vertical fixation locations and mean fixation durations. There were no main effects of 559 Condition, Road Section or interactions between Condition and Road Section for any of these eye 560 movement measures. 

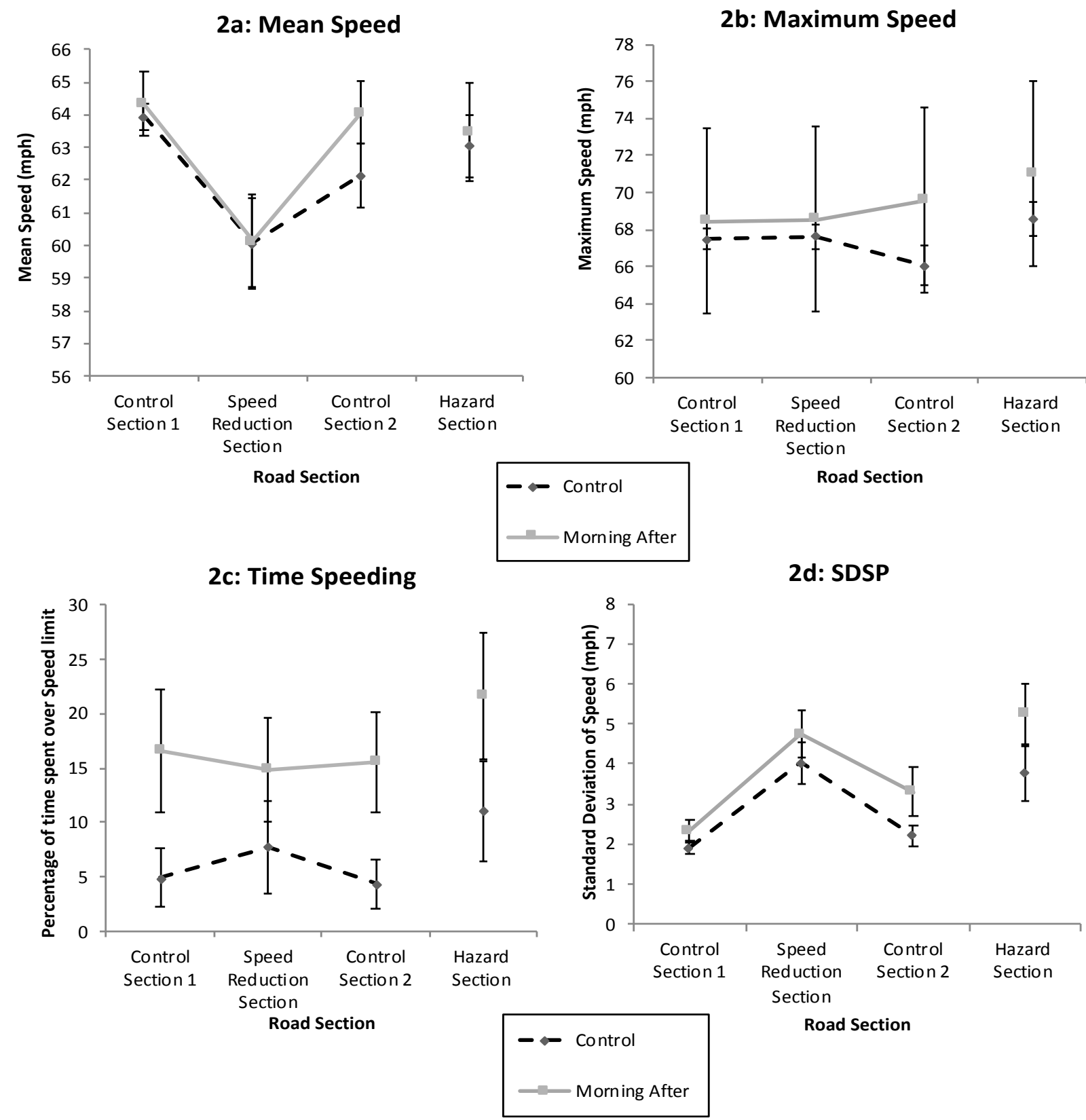

2d: SDSP

2e: Mean Acceleration

2f: SDLP

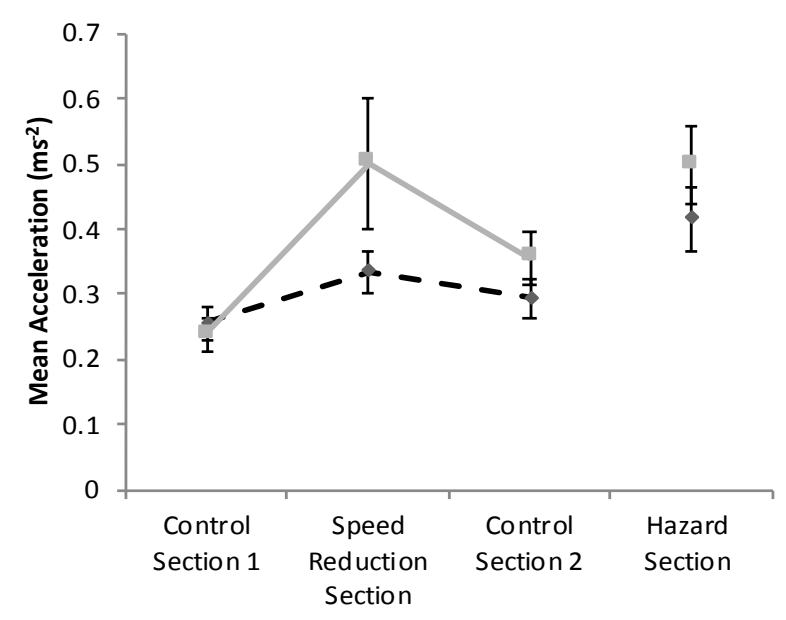

Road Section

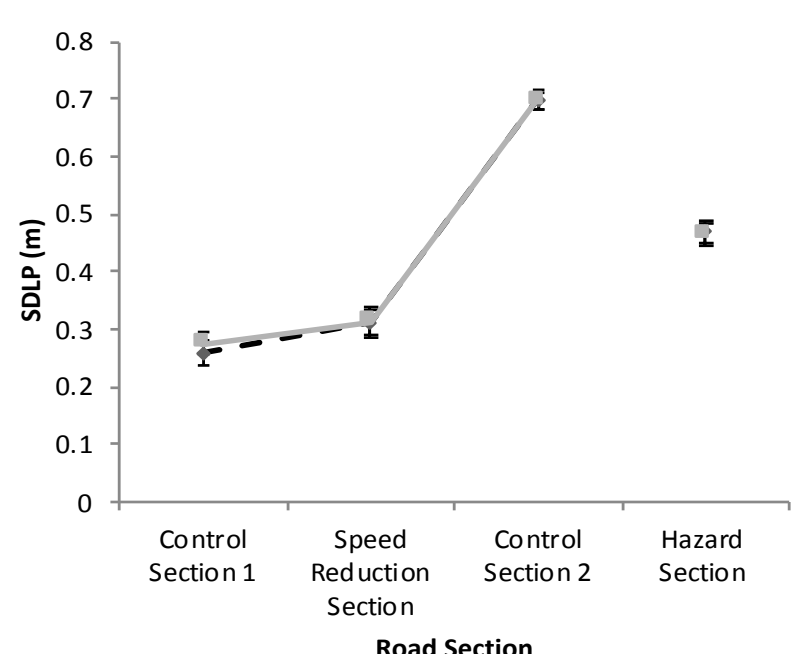

$561 \quad$ Figure 1: Shows the six measures of driving performance over the four sections of the drive, plotted separately for the control and morning after condition. Error bars show the one standard error above or below the mean. 


\section{Discussion.}

563 The current study investigated whether students were impaired at simulator driving the morning after a 564 night out social drinking, compared to a control night. The main finding of the study was that despite 565 twenty eight of the thirty participants being under the legal driving limit for breath alcohol content, 566 there was evidence of more dangerous driving the morning after compared to the control drive. It was 567 found that when participants completed the morning after drive, they tended to travel at higher maximum speeds, travel for a longer period of time over the speed limit, demonstrate a larger variance in speed, and have a marginal tendency to have a sharper change in speed. However, we did not find differences in drivers' visual attention measures while driving, nor differences in driving measures such as SDLP, suggesting that morning after alcohol consumption impaired our student drivers' judgements on the road, but not their attentiveness. The results of the study have important implications for road safety, as this study investigates a population which have been seen to commonly engage in social excessive drinking, and a sample who would be expected to engage in morning university commitments, which will often require travel to the university.

Firstly, it was found that there was a particular impairment on longitudinal vehicle control measures. The fact that drivers the morning after displayed significantly greater SDSP compared to control is consistent with a recent meta-analysis conducted on the effects of acute alcohol consumption on simulated driving (Irwin et al., 2017), recent studies investigating the effects of acute alcohol consumption on driving (Mets et al., 2011; McCartney et al., 2017), and research investigating the combined effects of alcohol and sleep deprivation on driving (Arnedt et al., 2001). Previous research from Verster et al., (2014a) did not find a difference in drivers' SDSP the morning after alcohol consumption compared to control however, they did find that SDSP (as well as SDLP and lapses of attention) was more pronounced as the driving task went on, with drivers performing worse on the second half of the driving task compared to the first. This finding was also supported by Verster and 587 Roth (2013) who investigated the progressive nature of driving impairment throughout a driving task 
in more depth, as well as Arnedt et al., (2001) in regards to sleep deprivation. Similarly, in the current

589 study it was found that although SDSP increased in the speed reduction zone as expected, it also

590 increased in control section two compared to control section one, suggesting that longitudinal

591 measures can get progressively worse, the longer the task.

In addition, it was found that drivers travelled at significantly higher maximum speeds in the morning after condition compared to the control condition, which is similar to previous findings from Charlton and Starkey (2013). This study found that drivers' higher maximum speeds were observed when participants were on the declining BAC limb compared to the same BAC on the ascending limb. This suggests that driving errors are not abolished on the apparent decreasing BAC stage (Charlton \& Starkey, 2015), therefore although many participants in the current study had low BrAC readings (the majority of our participants had a reading of 0 even in the Morning After condition), these driving 600 effects can still persist.

It was also found that drivers spent more time over the speed limit in the morning after condition compared to the control condition. Previous research has found that speeding is one of the most robust measures of alcohol impairment that affects driver choices directly (Christoforou, Karlaftis \& Yannis 2012), with the current study's result suggesting that this effect may extend to morning after driving when BrAC is declining, and therefore may be still likely to affect drivers' choices to violate road rules. It should also be highlighted that reduced speeds allow drivers extra time to adapt to changes and respond to risks in the environment, however, as drivers were travelling at higher speeds and breaking the speed limit more often, this allows for decreased time to respond to risks, resulting in an increased 610 risk of accident (Lenné et al., 2010). This accident risk could be made more severe in the Morning 611 After condition, as drivers had a marginal tendency to make sharper changes to their speed compared 612 to control, as previous research has suggested that abrupt braking can increase accident risk, 613 particularly seen with young novice drivers (McKnight \& McKnight, 2003). 
615 Surprisingly however, it was found that there were no differences in drivers' SDLP and drivers' visual 616 attention measures which included horizontal SD, vertical SD and mean fixation durations, despite 617 participants' recordings and reports of sleep being significantly lower and ratings of sleepiness being 618 significantly higher in the morning after condition compared to control. These results were inconsistent 619 with our original hypotheses, which proposed that driving the morning after would elicit behaviours 620 showing evidence of inattentiveness which included SDLP, reduced gaze deviations and longer mean 621 fixation durations. The current results contrast with results obtained by Verster et al., (2014a), and results which suggest that both fatigue (Otmani et al., 2005; Ingre et al., 2006) and alcohol (Helland et al., 2013; Irwin et al., 2017) commonly impair measures of visual attention exhibited by drivers' ability to maintain a steady lane position, as well as reducing gaze deviation and increasing mean fixation durations (Ogden \& Moskowitz, 2004; Ji \& Yang, 2002). However, inattentive and reckless driving have been argued to be fundamentally different, in which the former is associated with an inability to sustain focussed attention associated with self-regulation (Tay \& Knowles, 2004) and the latter a more volitional choice about dangerous driving behaviour (Kostermans et al., 2014).

In regards to drivers' mean speed, it was also found that there were no differences in the morning after condition and in the control condition, which is inconsistent with previous research (Mets et al., 2011;

631 McCartney et al., 2017) but consistent with other studies looking specifically at the morning after 632 effects of sleep deprivation (Peters et al., 1999) and alcohol hangover (Verster et al., 2014a). The lack 633 of difference in mean speed (and SDLP) in previous studies has been attributed to the sample, as they 634 used young healthy adults, who had no prolonged sleep deprivation prior to sleep induction paradigms, 635 therefore subtle highway measures may not be affected (Peters et al., 1999). In addition, the significant 636 reduction in mean speed in the speed reduction section suggests that the introduction of a new speed 637 limit influenced participants' behaviour, with some reduction in speed being evident in participants in 638 both conditions. This also supports the idea that drivers were not inattentive in regards to noticing the 639 road signs, but chose to travel at higher speeds than legally permitted. 
641 Finally, it was found that there was no significant difference in response time between the two 642 conditions towards the planned hazard. This was surprising given that alcohol is known to increase 643 drivers' response times (Summala \& Mikkola, 1994). However, from recent validation studies it is not 644 clear how reliable simulator environments are for higher behavioural control tasks such as hazard 645 avoidance (Engström, Johansson \& Östlund, 2005), with little previous evidence measuring this. This 646 hazard was also a planned single hazard, whereas multiple hazards may be encountered in real world 647 driving, requiring additional situational awareness.

649 So far, the current study's results suggest that students who drive the morning after a night out 650 drinking display impairments in driving judgement in terms of a greater willingness to violate road 651 rules, however, they do not demonstrate impairment in attention. Potential explanations regarding the 652 absence of effects in drivers' attentiveness will be discussed, alongside the potential interventions and 653 further research that could be investigated using the findings of the current study.

655 The lack of evidence for sustained attention measures such as SDLP and eye movements could be 656 related to the nature of our driving task. The current study used a 15-minute highway driving task, as 657 opposed to the one-hour drive used by Verster et al. (2014a). The short drive in the current study, 658 chosen as a means to avoid exacerbating the somatic symptoms participants may experience after 659 heavy drinking, may have not been long enough to elicit the effects of fatigue and thus inattention. 660 Previous research has highlighted changes in behaviour caused by fatigue are often only measurable 661 when high levels of fatigue are reached (Ingre et al., 2006), with previous evidence showing 662 detrimental effects to appear around 15 minutes into a driving task (Chapman et al., 1999). This 663 suggests that levels of fatigue in our participants may not have reached a necessary criterion level in 664 order for performance to be increasingly impaired, and may also explain why there were no effects on 665 SDLP and mean speed measures over the course of the drive. The two-fold nature of fatigued driving, 
including both the fatigue resulting from driving and the effects of fatigue from sleep deprivation the

667 night before (Crawford, 2007) could be argued to be present in the long drive used by Verster et al.

668 (2014a) but absent in the current study as the present 15-minute drive was short, interactive and

669 demanded changes in behaviour as a result of the variable speed limits. It is possible that our

670 participants may have also been partly able to counteract impairment throughout increased effort and

671 motivation to perform a 15-minute task (Verster \& Roth, 2013), as motivation has been seen to directly

672 influence drivers' ability to allocate attention to resources (Fuller, 2005).

673

674 Consequently, it could be argued that while Verster et al.'s (2014a) drive was long and monotonous

675 enough to induce fatigue itself, the current drive did not. The duration of the drive in the current study

676 is nonetheless reflective of journeys many students would regularly take in the morning after a night of

677 drinking, for instance from their home to university, and of the average duration of car journeys. Thus,

678 the results from the current study may be more generalisable to everyday driving in the UK than

679 previous research. This dissociation in results indicates perhaps that reckless driving is more of a

680 problem on short drives the morning after alcohol consumption, while inattentive driving may be

681 particularly important for longer drives in the morning after - an area appropriate for further

682 development in future research.

683

684 It is also possible that the naturalistic nature of the current study may explain why some of the results

685 found are inconsistent with previous research which had more controlled manipulations of alcohol

686 consumption and sleep deprivation. The current study was designed to replicate a common real-life

687 scenario, such as driving to work or university after a night out socialising, therefore there was an

688 unavoidable confound that participants would consume variable amounts of alcohol and have variable

689 sleep durations. Equally however, there are substantial advantages of conducting this experiment as a

690 naturalistic study, as this ecologically representative testing protocol can be argued to be more

691 generalisable to real life behaviour, with fewer research studies conducting morning after studies in a 
naturalistic way. As this study was measuring a situation which occurs regularly in university life, it was important the participants were a sample of young students who had planned to engage in social drinking, from a population known to commonly engage in drinking in excess (NUS, 2017) and who are over-represented in alcohol related crashes (DfT, 2017b).

The current study highlights the importance of appreciating that impairment in driving the morning after cannot be attributed to one particular cause or mechanism. The naturalistic nature of the investigation means that factors impairing students' morning after driving performance, such as alcohol or sleep deprivation were closely linked and not analytically separable in the current study. Despite this methodological limitation, it is apparent that the typical everyday combination of sleep deprivation and alcohol consumption can to have a selective detrimental effect on morning after driving performance, even on a relatively short drive. It is worth noting that this combination of impairments is likely to be common in real life driving even when drivers are still below the legal BAC. Attempts to fully disentangle the impairments associated with these two factors would require more controlled parameters with a less naturalistic design or a substantially larger naturalistic sample size with lower covariation between sleepiness and alcohol consumption.

In regards to practical implications, the results of the current study allow for interpretation of morning after driving impairment in terms of an accepted standard for safety. The current study highlights that alcohol induced driving impairments the morning after exist under the national speed limit and irrespective of legality to drive, indicating that drivers may be at risk on the road. It also highlights the difficulty with using absolute $\mathrm{BrAC}$ as a standard for safe driving. We found a detriment in performance the morning after drinking despite recorded BrAC being 0 for the majority of participants. It is also important to highlight that there was relatively little association between the selfreported units of alcohol consumed by students and their morning after breathalyser scores, despite around half of the participants reporting to have consumed more units in one night than the UK 
guidelines for weekly consumption. It is likely that the timing of alcohol consumption was critical in determining whether participants were over the limit the following morning but less important in determining whether driving was actually impaired.

There is also a concern regarding the dangers associated with sleep deprivation while driving, as there is presently no standard that exists against which sleep-related deficiencies can be judged. Although many countries set allowable BACs at the point that compromises safe performance, it is acknowledged that developing similar standards for fatigue to ensure that people with sleep deprivation are kept from risky behaviours such as driving is more challenging. One possibility is introducing educational programmes that highlight the impairments in driving that persist the morning after alcohol consumption and sleep deprivation, with the aim of preventing engagement in this behaviour. Alvaro et al. (2018) reported encouraging results for programmes raising awareness of the dangers of drowsy driving in young adults, therefore similar programmes could help apply these findings to the wider driving population.

The current study's results suggest that in a short highway driving task, students display morning after impairments in driving judgement in terms of a greater willingness to violate road rules however, do not demonstrate impairment in attention. To further investigate if the morning after elicits more impulsive behaviour generally, or it this is only limited to driving behaviours as reported in the current study, a similar naturalistic design but with the incorporation of behavioural task measuring response inhibition, such a go/no-go task (Fillmore, 2003) and sustained attention, such as the vigilance task by Bakan (1959) could be conducted to see if the same dissociative effect exists on simpler cognitive tasks. This might provide greater understanding of the mechanisms underlying the current pattern of impaired driving the morning after drinking. If inattention is measured on simple behavioural tasks, it could be postulated that the drive in the current study was not a sensitive enough measure of attention, or participants may have compensated for their fatigue by ensuring attention was maintained the morning after drinking. 


\subsection{Conclusions}

745 In conclusion, the current study makes a significant contribution to the current driving safety literature, 746 indicating that students who would typically be travelling to university after a night out demonstrate 747 dangerous judgements in driving behaviour the morning after. The main findings suggest that drivers 748 elicit reckless driving behaviours such as decreased adherence to speed limits, higher maximum 749 speeds, greater deviations in speeds and a marginal tendency to make sharper changes in their speed. 750 Despite this, drivers did not demonstrate any impairments in measures of attention, and more subtle 751 measures of driving behaviour such as SDLP and mean speed. These results suggest that despite 752 average BrAC being three times under the current national legal limit, the morning after effects of 753 alcohol are shown to elicit dangerous driving behaviour, with a greater willingness to violate road 754 rules, with no suggestions of inattentiveness while driving. It is highlighted that the current study's 755 results could be used to implement informative educational programmes about the impairments that 756 persist in morning after driving, making drivers aware that even when under the legal driving limit, 757 short distance drives the morning after drinking can elicit dangerous driving, with the potential of 758 longer drives leading to inattentiveness.

761 Acknowledgements:

762 The Nottingham Integrated Transport and Environment Simulation (NITES) facility provided the 763 simulator used to conduct this research.

\section{Declaration of Interests:}

766 None. 


\section{References.}

771

772

773

774

775

Åkerstedt, T., \& Kecklund, G. (2001). Age, gender and early morning highway accidents. Journal of Sleep Research, 10(2), 105-110

Alvaro, P. K., Burnett, N. M., Kennedy, G. A., Min, W. Y. X., McMahon, M., Barnes, M., ... \& Howard, M. E. (2018). Driver education: enhancing knowledge of sleep, fatigue and risky behaviour to improve decision making in young drivers. Accident Analysis \& Prevention, 112, $77-83$.

Arnedt, J. T., Wilde, G. J., Munt, P. W., \& MacLean, A. W. (2001). How do prolonged wakefulness and alcohol compare in the decrements they produce on a simulated driving task? Accident Analysis \& Prevention, 33(3), 337-344.

Bakan, P. (1959). Extraversion-introversion and improvement in an auditory vigilance task. British Journal of Psychology, 50(4), 325-332.

Banks, S., Catcheside, P., Lack, L., Grunstein, R. R., \& McEvoy, R. D. (2004). Low levels of alcohol impair driving simulator performance and reduce perception of crash risk in partially sleep deprived subjects. Sleep, 27, 1063-1067.

Buchner, A., Faul, F., \& Erdfelder, E. (1992). G Power: a priori, post-hoc, and compromise power analyses for the Macintosh. Bonn, Germany: Bonn University.

Brookhuis KA, De Waard D, Samyn N (2004) Effects of MDMA (ecstacy), and multiple drug use on (simulated) driving performance and traffic safety. Psychopharmacology 173:440-445 
797 Carlson, W. L. (1972). Alcohol usage of the nighttime driver. Journal of Safety Research, 4(1), 12-25. 798

799 Chapman, P., Ismail, R., Avellano, T., \& Underwood, G. (1999). Time-gaps while driving. In G.B. 800 Grayson (Ed.) Behavioural Research In Road Safety IX, pp.188-200. Transport Research $801 \quad$ Laboratory, Crowthorne: UK. PA3524/99.

803 Charlton, S. G., \& Starkey, N. J. (2013, August). Drunker than you think: Delayed performance impairment from moderate amounts of alcohol. In InAustralasian Road Safety Research Policing Education Conference.

Charlton, S. G., \& Starkey, N. J. (2015). Driving while drinking: performance impairments resulting 808 from social drinking. Accident Analysis \& Prevention, 74, 210-217.

Christoforou, Z., Karlaftis, M. G., \& Yannis, G. (2012). Effects of alcohol on speeding and road positioning of young drivers: Driving simulator study. Transportation Research

814 Crawford, A. (1961). Fatigue and driving. Ergonomics, 4(2), 143-154.

816 Cromer, J. R., Cromer, J. A., Maruff, P., \& Snyder, P. J. (2010). Perception of alcohol intoxication shows acute tolerance while executive functions remain impaired. Experimental and Clinical Psychopharmacology, 18(4), 329. 
820 Degia, A., Meadows, R., Johnsen, S., Dixon, P., Hindmarch, I., \& Boyle, J. (2006). Investigation into the suitability of a portable psychometric device to be used in the field: an illicit drugs field investigation. Journal of Clinical Forensic Medicine, 13(5), 242-246.

Department for Transport (2017a). Reported road casualties in Great Britain, final estimates involving illegal alcohol levels: 2016 report. Retrieved from: https://assets.publishing.service.gov.uk/government/uploads/system/uploads/attachment_data/fi le/732650/drink-drive-final-estimates-2016.pdf

Department for Transport. (2017b). Reported Road Casualties. Great Britain: 2016. National Statistics. Retrieved from: https://www.gov.uk/government/statistics/reported-road- casualties-greatbritain-annual-report-2016

Department for Transport. (2017c). National Travel Survey: 2016. National Statistics. Retrieved from: https://www.gov.uk/government/statistics/national-travel-survey- 2016 a common attentional mechanism. Vision Research, 36(12), 1827-1837.

Deubel, H., \& Schneider, W. X. (1996). Saccade target selection and object recognition: Evidence for

843 Fillmore, M. T. (2003). Drug abuse as a problem of impaired control: current approaches and findings. Behavioral and Cognitive Neuroscience Reviews, 2(3), 179-197. 
846 Fillmore, M. T., Blackburn, J. S., \& Harrison, E. L. (2008). Acute disinhibiting effects of alcohol as a factor in risky driving behavior. Drug and Alcohol Dependence, 95(1-2), 97-106.

Fogarty, J. N., \& Vogel-Sprott, M. (2002). Cognitive processes and motor skills differ in sensitivity to alcohol impairment. Journal of Studies on Alcohol, 63(4), 404-411.

Fuller, R. (2005). Towards a general theory of driver behaviour. Accident Analysis \& Prevention, 37(3), 461-472.

\section{8}

Gharagozlou, F., Mazloumi, A., Saraji, G. N., Nahvi, A., Ashouri, M., \& Mozaffari, H. (2015). Correlation between driver subjective fatigue and bus lateral position in a driving simulator. Electronic Physician, 7(4), 1196-1204.

\section{3}

864 Hershner, S. D., \& Chervin, R. D. (2014). Causes and consequences of sleepiness among college students. Nature and Science of Sleep, 6, 73-84. simulated driving performance and blink duration: examining individual differences. Journal of 
Irwin, C., Iudakhina, E., Desbrow, B., \& McCartney, D. (2017). Effects of acute alcohol consumption on measures of simulated driving: a systematic review and meta- analysis. Accident Analysis \& Prevention, 102, 248-266.

Ji, Q., \& Yang, X. (2002). Real-time eye, gaze, and face pose tracking for monitoring driver vigilance. Real-time Imaging, 8(5), 357-377.

Jongen, S., Perrier, J., Vuurman, E. F., Ramaekers, J. G., \& Vermeeren, A. (2015). Sensitivity and validity of psychometric tests for assessing driving impairment: effects of sleep deprivation. PLoS ONE, 10(2), e0117045.

Kennedy, R.S., Lane, N.E., Berbaum, K.S., \& Lilienthal, M.G. (1993). Simulator Sickness Questionnaire: An enhanced method for quantifying simulator sickness. International Journal of Aviation Psychology, 3(3), 203-220.

Kostermans, E., Stoolmiller, M., de Leeuw, R. N., Engels, R. C., \& Sargent, J. D. (2014). Exposure to movie reckless driving in early adolescence predicts reckless, but not inattentive driving. PloS ONE, 9(12), e113927.

Lenné, M. G., Dietze, P. M., Triggs, T. J., Walmsley, S., Murphy, B., \& Redman, J. R. (2010). The effects of cannabis and alcohol on simulated arterial driving: influences of driving experience and task demand. Accident Analysis \& Prevention, 42(3), 859-866.

Ling, J., Stephens, R., \& Heffernan, T. M. (2010). Cognitive and psychomotor performance during alcohol hangover. Current Drug Abuse Reviews, 3(2), 80-87. 
MacDonald, S. (1999). Unrecorded alcohol consumption in Ontario, Canada: estimation procedures and research implications. Drug and Alcohol Review, 18(1), 21-29.

Marczinski, C. A., \& Fillmore, M. T. (2009). Acute alcohol tolerance on subjective intoxication and simulated driving performance in binge drinkers. Psychology of Addictive Behaviors, 23(2), 238.

Marczinski, C. A., Harrison, E. L., \& Fillmore, M. T. (2008). Effects of alcohol on simulated driving and perceived driving impairment in binge drinkers. Alcoholism: Clinical and Experimental Research, 32(7), 1329-1337.

Martiniuk, A. L., Senserrick, T., Lo, S., Williamson, A., Du, W., Grunstein, R. R., ... \& Ivers, R. Q. (2013). Sleep-deprived young drivers and the risk for crash: the DRIVE prospective cohort study. JAMA Paediatrics, 167(7), 647-655.

McCartney, D., Desbrow, B., \& Irwin, C. (2017). Using alcohol intoxication goggles (Fatal Vision® goggles) to detect alcohol related impairment in simulated driving. Traffic Injury Prevention, $18(1), 19-27$.

McKinney, A., \& Coyle, K. (2004). Next day effects of a normal night's drinking on memory and psychomotor performance. Alcohol and Alcoholism, 39(6), 509-513.

Mc Kinney, A., \& Coyle, K. (2005). Alcohol hangover effects on measures of affect the morning after a normal night's drinking. Alcohol and Alcoholism, 41(1), 54-60. 
922 McKinney, A., Coyle, K., \& Verster, J. (2012). Direct comparison of the cognitive effects of acute alcohol with the morning after a normal night's drinking. Human Psychopharmacology: Clinical and Experimental, 27(3), 295-304.

925

McKnight, A. J., \& McKnight, A. S. (2003). Young novice drivers: careless or clueless?. Accident Analysis \& Prevention, 35(6), 921-925. Effects of alcohol on highway driving in the STISIM driving simulator. Human Psychopharmacology: Clinical and Experimental, 26(6), 434-439.

National Health Service. (2018). Alcohol Support. Retreived from: https://www.nhs.uk/livewell/alcohol-support/calculating-alcohol-units/

National Union of Students. (2017). Students and Alcohol Survey. NUS Alcohol Impact. Retrieved Traffic Injury Prevention, 5(3), 185-198.

942 O’Hanlon, J. F., \& Kelley, G. R. (1977). Comparison of performance and physiological changes 943 between drivers who perform well and poorly during prolonged vehicular operation. Vigilance, 3, 87-109. 
Orzel-Gryglewska, J. (2010). Consequences of sleep deprivation. International Journal of Occupational Medicine and Environmental Health, 23(1), 95-114. doi: 10.2478/v10001-0100004-9

Otmani, S., Pebayle, T., Roge, J., \& Muzet, A. (2005). Effect of driving duration and partial sleep deprivation on subsequent alertness and performance of car drivers. Physiology \& Behavior, 84(5), 715-724.

Otmani, S., Pebayle, T., Roge, J., \& Muzet, A. (2005). Effect of driving duration and partial sleep deprivation on subsequent alertness and performance of car drivers. Physiology \& Behavior, 84(5), 715-724.

Peters, R. D., Wagner, E. K., Alicandri, E., Fox, J. E., Thomas, M. L., Thorne, D. R., ... \& Balwinski, S. M. (1999). Effects of partial and total sleep deprivation on driving performance. Public Roads, 62(4).

Prat, G., Adan, A., Pérez-Pàmies, M., \& Sànchez-Turet, M. (2008). Neurocognitive effects of alcohol hangover. Addictive Behaviors, 33(1), 15-23.

RAC. (2017). Report on Motoring 2017. Feeling the Squeeze. Retrieved from: https://www.rac.co.uk/pdfs/report-on-motoring/rac_rom_2017.pdf

Reed, M. P., \& Green, P. A. (1999). Comparison of driving performance on-road and in a low-cost simulator using a concurrent telephone dialling task. Ergonomics, 42(8), 1015-1037. 
Rogé, J., Pébayle, T., El Hannachi, S., \& Muzet, A. (2003). Effect of sleep deprivation and driving duration on the useful visual field in younger and older subjects during simulator driving. Vision Research, 43(13), 1465-1472.

Shahid, A., Wilkinson, K., Marcu, S., \& Shapiro, C. M. (2012). Stanford Sleepiness Scale (SSS). In STOP, THAT and One Hundred Other Sleep Scales (pp. 369-370). Springer New York.

Starkey, N. J., \& Charlton, S. G. (2014). The effects of moderate alcohol concentrations on driving and cognitive performance during ascending and descending blood alcohol concentrations. Human Psychopharmacology: Clinical and Experimental, 29(4), 370- 383.

Stephens, R., Ling, J., Heffernan, T. M., Heather, N., \& Jones, K. (2008). ReviewA review of the literature on the cognitive effects of alcohol hangover. Alcohol and Alcoholism, 43(2), 163-170.

Summala, H., \& Mikkola, T. (1994). Fatal accidents among car and truck drivers: effects of fatigue, age, and alcohol consumption. Human Factors, 36(2), 315-326.

Tiplady, B., Degia, A., \& Dixon, P. (2005). Assessment of driver impairment: Evaluation of a twochoice tester using ethanol. Transportation Research Part F: Traffic Psychology and Behaviour, 8(4-5), 299-310.

Tay, T., \& Knowles, D. (2004). Driver inattention: drivers' perception of risks and compensating behaviours. IATSS research, 28(1), 89-94.

Tiplady, B., \& Degia, A. (2004, August). Evaluation of tests of driver impairment suitable for roadside use. In 17th International Conference on Alcohol, Drugs, and Traffic Safety, Glasgow. 
998 Törnros, J., \& Laurell, H. (1991). Acute and hang-over effects of alcohol on simulated driving performance. Blutalkohol, 28(1), 24-30

1000

1001 Velichkovsky, B. M., Rothert, A., Kopf, M., Dornhöfer, S. M., \& Joos, M. (2002). Towards an express-diagnostics for level of processing and hazard perception. Transportation Research Part F: Traffic Psychology and Behaviour, 5(2), 145-156.

1004

Verster, J. C., Bervoets, A. C., de Klerk, S., Vreman, R. A., Olivier, B., Roth, T., \& Brookhuis, K. A. (2014a). Effects of alcohol hangover on simulated highway driving performance. Psychopharmacology, 1-10.

1008

1009 Verster, J. C., Mackus, M., van de Loo, A. J., Garssen, J., \& Scholey, A. (2017). The breathtaking 1010 truth about breath alcohol readings of zero. Addictive Behaviors, 70, 23- 26.

1012 Verster, J. C., \& Roth, T. (2011). Standard operation procedures for conducting the on-the-road 1013 driving test, and measurement of the standard deviation of lateral position (SDLP). International Journal of General Medicine, 4, 359.

1016 Verster, J. C., \& Roth, T. (2013). Vigilance decrement during the on-the-road driving tests: The importance of time-on-task in psychopharmacological research. Accident Analysis \& 1019

Verster, J. C., \& Roth, T. (2014). Effects of central nervous system drugs on driving: speed variability versus standard deviation of lateral position as outcome measure of the on- the-road driving test. Human Psychopharmacology: Clinical and Experimental, 29(1), 19-24. 
1025 Verster, J. C., Stephens, R., Penning, R., Rohsenow, D., McGeary, J., Levy, D., ... \& Batty, G. D. (2010). The alcohol hangover research group consensus statement on best practice in alcohol hangover research. Current Drug Abuse Reviews, 3(2), 116-126.

Verster, J. C., Van Der Maarel, M. A., McKinney, A., Olivier, B., \& De Haan, L. (2014b). Driving during alcohol hangover among Dutch professional truck drivers. Traffic Injury Prevention, $15(5), 434-438$.

Weafer, J., \& Fillmore, M. T. (2012). Acute tolerance to alcohol impairment of behavioral and cognitive mechanisms related to driving: drinking and driving on the descending limb. Psychopharmacology, 220(4), 697-706.

van Schrojenstein Lantman, M., van de Loo, A. J., Mackus, M., \& Verster, J. C. (2016). Development of a definition for the alcohol hangover: consumer descriptions and expert consensus. Current Drug Abuse Reviews, 9(2), 148-154.

1045 Weafer, J., \& Fillmore, M. T. (2016). Low-dose alcohol effects on measures of inhibitory control, 1046 delay discounting, and risk-taking. Current Addiction Reports, 3(1), 75-84. 
1048 Williamson, A. M., \& Feyer, A. M. (2000). Moderate sleep deprivation produces impairments in 1049 cognitive and motor performance equivalent to legally prescribed levels of alcohol intoxication. $1050 \quad$ Occupational and Environmental Medicine, 57(10), 649-655.

1051

1052 World Health Organisation. (2016). Global Status Report on Road Safety 2015. Geneva. Retrieved 1053 from: http://www.who.int/violence_injury_prevention/road_safety_status/2015/en/ 